\title{
Resistive contribution in electrical-switching experiments with antiferromagnets
}

\author{
Tristan Matalla-Wagner $\odot,{ }^{1, *}$ Jan-Michael Schmalhorst,${ }^{1}$ Günter Reiss, ${ }^{1}$ Nobumichi Tamura, ${ }^{2}$ and Markus Meinert $\oplus^{3, \dagger}$ \\ ${ }^{1}$ Center for Spinelectronic Materials and Devices, Bielefeld University, Universitätsstraße 25, D-33501 Bielefeld, Germany \\ ${ }^{2}$ Advanced Light Source, Lawrence Berkeley National Laboratory, Berkeley, California 94720, USA \\ ${ }^{3}$ Department of Electrical Engineering and Information Technology, Technical University Darmstadt, D-64283 Darmstadt, Germany
}

(Received 22 October 2019; accepted 22 June 2020; published 15 July 2020)

\begin{abstract}
Recent research demonstrated the electrical switching of antiferromagnets via intrinsic spin-orbit torque or the spin Hall effect of an adjacent heavy metal layer. The electrical readout is typically realized by measuring the transverse anisotropic magnetoresistance at planar cross- or star-shaped devices with four or eight arms, respectively. Depending on the material, the current density necessary to switch the magnetic state can be large, often close to the destruction threshold of the device. We demonstrate that the resulting electrical stress changes the film resistivity locally and thereby breaks the fourfold rotational symmetry of the conductor. This symmetry breaking due to film inhomogeneity produces signals, that resemble the anisotropic magnetoresistance and is experimentally seen as a "saw-tooth"-shaped transverse resistivity. This artifact can persist over many repeats of the switching experiment and is not easily separable from the magnetic contribution. We discuss the origin of the artifact, elucidate the role of the film crystallinity, and propose approaches how to separate the resistive contribution from the magnetic contribution.
\end{abstract}

DOI: 10.1103/PhysRevResearch.2.033077

\section{INTRODUCTION}

The possibility to switch the magnetic order of antiferromagnets (AFM) with electric currents has been predicted about five years ago [1]. It was theoretically shown that materials of certain symmetry can exhibit staggered nonequilibrium spin polarization. If this coincides with the crystallographic positions of the staggered magnetic moments, a net torque on the antiferromagnetic order arises, which enables its current-driven reorientation. While the effect was predicted for $\mathrm{Mn}_{2} \mathrm{Au}$, its first realization was demonstrated with CuMnAs [2]. Later, the Néel-order switching was also shown for $\mathrm{Mn}_{2} \mathrm{Au}$ films [3-5]. A greater variety of AFM can be switched using heterostructures that utilize the spin Hall effect (SHE) in an adjacent heavy metal (HM) like Pt to exert an antidamping torque on the Néel-order [6-11]. The reorientation can be realized with cross-shaped planar devices, where electrical pulses driven through the orthogonal lines allow for a reproducible switching of the Néelorder by $90^{\circ}$. In AFM/HM heterostructures, there are two possible readout mechanisms with identical symmetry. The spin-polarized current in the HM is partially absorbed by the AFM, which depends on the relative orientation of the polarization and the magnetic moments in the AFM. This gives rise to the spin Hall magnetoresistance (SMR). The

\footnotetext{
*tristan@physik.uni-bielefeld.de

†markus.meinert@tu-darmstadt.de
}

Published by the American Physical Society under the terms of the Creative Commons Attribution 4.0 International license. Further distribution of this work must maintain attribution to the author(s) and the published article's title, journal citation, and DOI. second mechanism is the anisotropic magnetoresistance (AMR). In both cases, typically $\rho_{x x}>\rho_{y y}$ for the current flowing parallel or perpendicular to the magnetic moments is found. In single layer switching experiments, only the AMR is available for electrical readout. To observe the rather small resistivity change associated with the AMR or SMR, it is favorable to measure in transverse geometry. For a sense current density $j$, the associated transverse electric field is given by $E_{\perp}=j\left(\rho_{x x}-\rho_{y y}\right) \sin (2 \varphi) / 2$, where $\varphi$ is the angle between sense current density and magnetic moments. The experiment is performed by recording the transverse voltage $V_{\perp}=R_{\perp} I_{\mathrm{S}}$ with a fixed sense current $I_{\mathrm{s}}$ in a device as sketched in Fig. 1(a). In this type of experiments, Cheng et al. identified a "saw-tooth"-shaped contribution in $R_{\perp}$ measurements arising from the Pt layer for large current density [12], which is of nonmagnetic origin. It was found to be larger than the magnetic contribution to the transverse electrical response in some cases. Recently, Chiang et al. obtained similar results and proposed a model to explain their finding [13]. They suggest that the high pulse currents lead to lateral temperature gradients and, thus, to Seebeck voltages which contribute to the $V_{\perp}$ measurement. According to Cheng's interpretation, these voltages are of significant magnitude and persist over hours.

In the present manuscript, we investigate this nonmagnetic contribution to the electrical response of the starshaped structures and discuss a mechanism that is different from Chiang's approach and capable to explain the observed behavior on a semi-quantitative level. We chose paramagnetic $\mathrm{Nb}$ films as a model system. Two different types of films are compared, grown either at room temperature (polycrystalline) or at high temperature (epitaxial). We analyze the $R_{\perp}$ response to current pulses and inspect the starshaped devices in operando by x-ray microdiffraction $(\mu \mathrm{XRD})$ and scanning electron microscopy (SEM). Our findings are 
(a)
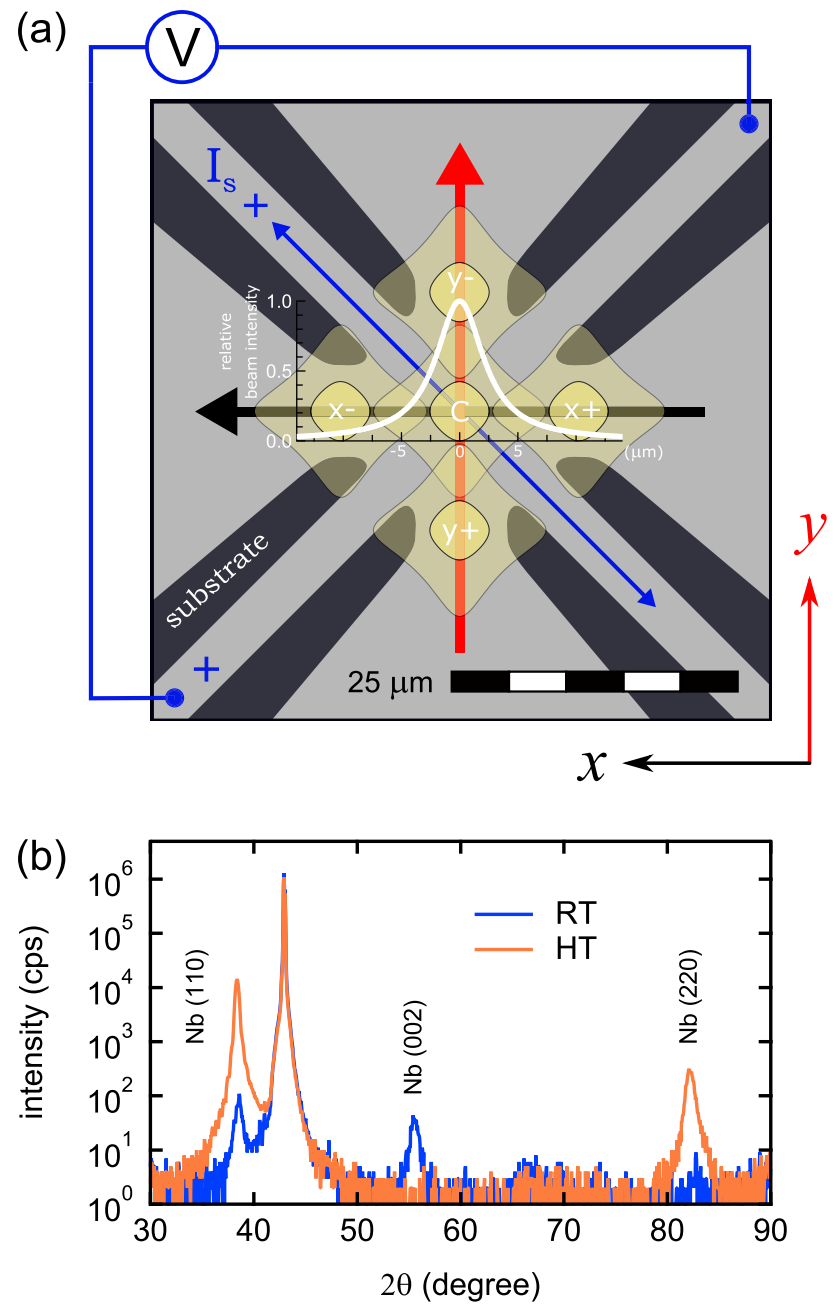

FIG. 1. Sample preparation. (a) Schematic of the device. Current pulses can be applied to the pulse lines parallel to the $x$ or $y$ axis. The red and black arrows indicate the conventional current direction for positive polarity. An AC sensing current $I_{\mathrm{s}}$ is applied to a probe line and the voltage $V$ is measured perpendicular to $I_{\mathrm{s}}$. The pulse and probe lines have a width of $8 \mu \mathrm{m}$ and $4 \mu \mathrm{m}$, respectively. Yellow areas show the positions of the x-ray spot for $\mu$ XRD measurements and the nomenclature for the positions is written in white. The two contours correspond to $50 \%$ and $10 \%$ intensity. A Lorentzian intensity profile highlights that the beam intensity at the constrictions is nonzero for the beam being centered at position C. (b) XRD measurements of unpatterned $\mathrm{Nb}$ films grown at room temperature (RT) and $400{ }^{\circ} \mathrm{C}$ (high temperature, HT) directly after deposition.

consistent with the data obtained from the transport experiments presented in Refs. [12,13], where a change of $R_{\perp}$ is observed in Pt films. However, we interpret this observation as a local variation of the film resistivity which gives rise to a $R_{\perp}$ change $\Delta R_{\perp}$ with alternating sign for the different pulsing directions. Calculations using a finite-element-method (FEM) show that the effect magnitude is in quantitative agreement with this explanation. Furthermore we see that the sign of $\Delta R_{\perp}$ can change within one experiment highlighting that at least two processes of nonmagnetic origin can be present in such experiments, related to locally decreasing or increasing resistivities.

\section{SAMPLE CHARACTERISTICS AND EXPERIMENTAL SETUP}

Samples of $\mathrm{MgO}(001)$ / $\mathrm{Nb} 25 \mathrm{~nm} / \mathrm{Si} 2 \mathrm{~nm}$ were grown by magnetron sputtering at room temperature (RT) and at $400^{\circ} \mathrm{C}$ (high temperature, HT). The Si capping prevents oxidation of the films. The film quality is characterized by $\mathrm{x}$-ray diffraction (XRD). The RT sample shows no preferred growth direction, whereas the HT sample grows in (110) direction, see Fig. 1(b). The resistivities are $\rho_{\mathrm{RT}}=27.4 \mu \Omega \mathrm{cm}$ and $\rho_{\mathrm{HT}}=23.5 \mu \Omega \mathrm{cm}$, determined by four-point measurements on the films. Finally, the films are patterned to star-shaped devices as depicted in Fig. 1(a) by photolithography and wirebonded into IC packages. On each sample, half of the devices have their pulse lines $x(y)$ aligned parallel to the [100] ([010]) direction of the substrate, the other half of the devices are rotated by $45^{\circ}$.

The experimental procedure for the electrical experiments is similar to our experiment on Néel-order switching in CuMnAs, see Ref. [14]. We apply $n$ bursts of current pulses to our structure in one of two orthogonal directions $x$ and $y$ [cf. Fig. 1(a)]. The pulses have a length of $\Delta t=1-10 \mu \mathrm{s}$ and a duty cycle of $10^{-2}$. The number of pulses per burst is not constant in this work but typically a burst consists of around 100 pulses. After each burst, we measure $R_{\perp}$ with a lock-in amplifier. We also observe the evolution of $R_{\perp}$ after the burst sequence during a relaxation-phase. The pulseline resistances $R_{x}$ and $R_{y}$ are measured prior to the burst sequence with a Keithley 2000 multimeter to set the voltage output of the arbitrary waveform generator accordingly. The experiments are additionally performed with simultaneous $\mu X R D$ measurements or SEM imaging. The $\mu$ XRD investigation was conducted at beamline 12.3.2 of the Advanced Light Source (ALS), Berkeley, California, USA [15]. We measure diffraction patterns with a PILATUS $1 \mathrm{M}$ detector and an integration time of $10-30 \mathrm{~s}$ for the beam located on the constrictions of each pulseline and in the center of the device. The spot is rectangular with a Lorentzian intensity profile that expands primarily perpendicular to the slit edges that form the spot. The full width at half maximum reads $5 \mu \mathrm{m}$ in $x$ and $y$ direction, as depicted in Fig. 1(a). Due to the long tails associated with a Lorentzian intensity profile, a fraction of the beam will hit the constrictions of the device even if positioned in the center. $\mu$ XRD measurements are always taken in the same order, i.e., $\{\mathrm{C}, x-, y-, x+, y+\}$. The photon energy $h v$ is tuned to suit the diffraction conditions of selected peaks. FEM calculations are performed with version 4.2 of the FEMM software package [16,17].

\section{RESULTS}

A pulsing experiment performed with $\mathrm{Nb}$ thin films is presented in Fig. 2. Panel (a) shows the change of $R_{\perp}$ for the RT sample with a current density of $j=7.5 \times 10^{11} \mathrm{~A} / \mathrm{m}^{2}$ applied alternating to the $x$ and $y$ pulseline. The respective relaxation is observed for $T_{\text {relax }}=60 \mathrm{~s}$. We observe a significant change of $R_{\perp}$ during pulsing, whereas $R_{\perp}$ remains stable during the relaxation. Initially, pulsing along $x$ increases $R_{\perp}$ while pulsing along $y$ decreases $R_{\perp}$. The amplitude of this "saw-tooth" signal decreases with each repeat and vanishes 

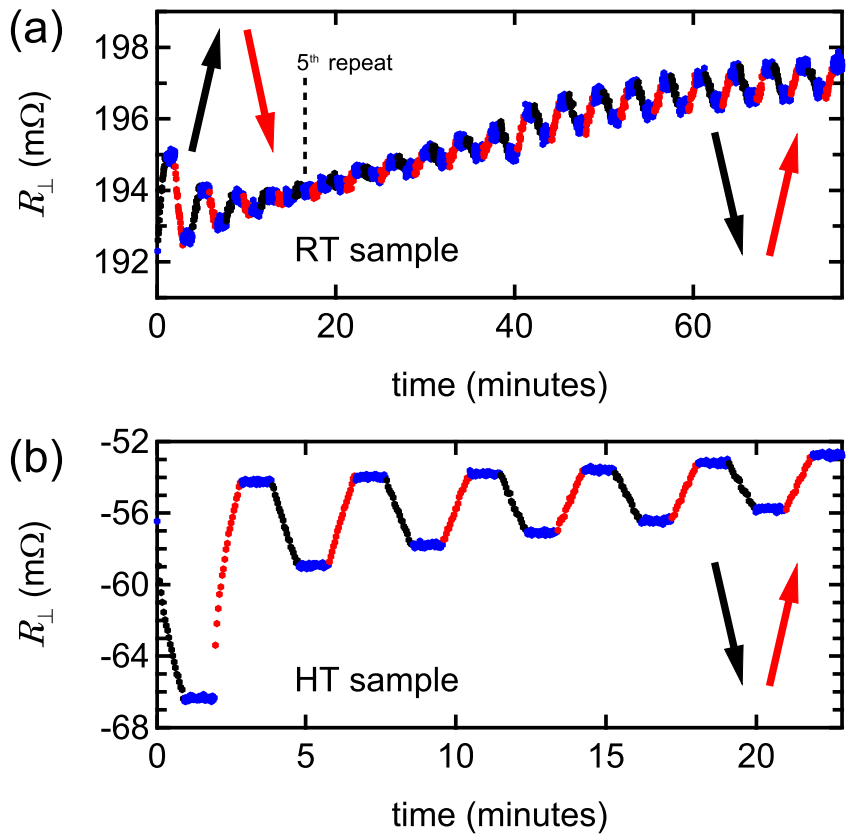

FIG. 2. Pulsing experiment. (a) Pulses with $j=$ $7.5 \times 10^{11} \mathrm{~A} / \mathrm{m}^{2}$ and $\Delta t=10 \mu \mathrm{s}$ are applied to a device on the RT sample with $x \| \mathrm{MgO}[100]$. The colors of the data points match the arrows in Fig. 1(a) to identify the pulsing and the relaxation phase. The arrows here accentuate the sign of $d R_{\perp} / d t$ that correlates with a certain pulsing direction. This sign inverts after the fifth repeat. (b) A similar measurement performed at the HT sample with $j=11.0 \times 10^{11} \mathrm{~A} / \mathrm{m}^{2}$ and $\Delta t=2 \mu \mathrm{s}$. The offset to $R_{\perp}$ in (a) and (b) arises from imperfect lithography, i.e., the transverse voltage pick up lines are not perfectly in line.

around the fifth repeat. The following repeats show an inverted sign of the response with virtually constant amplitude. An overall trend towards higher values of $R_{\perp}$ is present which is most likely related to asymmetries arising from imperfect lithography. The polarity of the pulse current has no impact on the signal (not shown). Panel (b) of Fig. 2 shows an equivalent measurement for the HT sample. The experimental parameters have been adjusted to find an electrical response of similar magnitude. From a current density variation we find, that the HT sample only shows one sign of transverse resistance change. The degradation of the transverse resistance amplitude slows down for successive repeats. We observe no qualitative difference in behavior between devices with $x \|$ $\mathrm{MgO}[100]$ or $x \| \mathrm{MgO}[110]$. Note that the result of this initial experiment is solely to determine which signs of transversal resistance change are present in the RT and HT samples, respectively.

In the following, we investigate a fresh device on the RT sample and take SEM images after each pulsing experiment with the device grounded while taking the image. Here, we primarily pulse along $x$ and use a single pulse sequence in $y$ direction only to demonstrate that the sign of $\Delta R_{\perp}$ depends on the pulsing direction as before. The $R_{\perp}$ response of the system and the values of $R_{x}$ taken intermittently are plotted in Figs. 3(a) and 3(b), respectively. For $j \leqslant 6.0 \times 10^{11} \mathrm{~A} / \mathrm{m}^{2}$, we neither see a transverse response nor a change of $R_{x}$. Starting with $j=6.5 \times 10^{11} \mathrm{~A} / \mathrm{m}^{2}$, we observe a response with $\Delta R_{\perp}>0$ and a decrease of $R_{x}$. Further increasing $j$, enhances the transverse response and the resistance change $\Delta R_{x}$ alike. Pulsing in $y$ direction changes the sign of $\Delta R_{\perp}$ and has no impact on $R_{x}$. This cycling capability is present at any current density (not shown). For successive bursts in the same direction, $\Delta R_{\perp}$ and $\Delta R_{x}$ seem to saturate until $j$ is increased again. At $j=8.0 \times 10^{11} \mathrm{~A} / \mathrm{m}^{2}$, we observe a crossover between reducing and increasing $R_{x}$, where the change of the transverse response also changes sign. This change in sign becomes more apparent for even higher $j$ until a response similar to Fig. 2(b) is obtained for $j=9.0 \times 10^{11} \mathrm{~A} / \mathrm{m}^{2}$. The effect size greatly enhances for $j=9.5 \times 10^{11} \mathrm{~A} / \mathrm{m}^{2}$ as seen in Figs. 3(c) and 3(d). For this current density, we observe that the transverse response intermittently has a positive sign, while the overall trend is negative. $R_{x}$ increases with each successive experiment until the device breaks. We were not able to resolve any change of the device in SEM images for $j \leqslant 9.0 \times 10^{11} \mathrm{~A} / \mathrm{m}^{2}$. However, from the first sequence at $j=9.5 \times 10^{11} \mathrm{~A} / \mathrm{m}^{2}$ until the destruction of the device we see clear changes arising at the lower left constriction of the structure. An exemplary image taken after the second repeat with highest $j$ is shown in Fig. 3(e). With the first burst of the fifth repeat the device breaks [cf. Fig. 3(f)] with droplets of material moving in electron flow direction.

We identified a correlation between $R_{x}$ and $R_{\perp}$ where the physical origin of this resistance change needs to be clarified. In the following, we attempt to investigate whether local annealing due to the Joule heating may contribute to the observed behavior. To this end, we perform $\mu$ XRD observations of selected X-ray diffraction peaks while performing pulsing experiments. Exemplarily, we show 2D diffractograms in Fig. 4. The positions of the x-ray focus are shown in Fig. 1(a). During the experiment, we do not observe any changes of the peak positions. To determine the crystallinity of the samples, we define regions of interest (ROI) around the peak positions and calculate the integrated intensity $I_{n \text {,raw }}=\sum^{\text {pixel }} i_{\text {pixel }}$, with $i_{\text {pixel }}$ being the counts of each pixel and $n$ being the experiment index. The x-ray intensity of the beamline varies slightly over time, so we normalize $I_{n \text {,raw }}$ to the background signal $I_{\mathrm{bg}}$ which is the average pixel brightness calculated from an ROI that contains only diffuse scattering and fluorescence signals. In Fig. 5, we plot the normalized peak intensity $I_{n}=I_{n, \text { raw }} / I_{\mathrm{bg}}$ divided by $I_{0}$ against the experiment index, which is essentially a number raised with each pulsing-relaxation sequence containing no information about $j$ or the pulsing direction. Because of the noisy signal, the measured value of $I_{n=0}$ is not suitable for normalization of the following measurements. Instead, we determine the normalization factor $I_{0}$ by applying a linear fit individually to each data set $\left(n, I_{n}\right)$. Figures $5(\mathrm{a})$ and 5(b) show the data measured at the RT and HT samples, respectively. The error bars are calculated as $\Delta I_{n}=\sqrt{I_{n}}$ following Poisson statistics which implies the assumption of a perfect detector. This methodology underestimates the error but allows to compare data sets of varying peak intensities. Note that the data sets shown in Fig. 5(a) have varying errors which change systematically with measurement position and observed peak. We interpret this apparent inconsistency as an artifact due to the movement of the sample stage in combination with a measurement performed at imperfect diffraction condition. For the RT sample, $h v$ is chosen to capture 

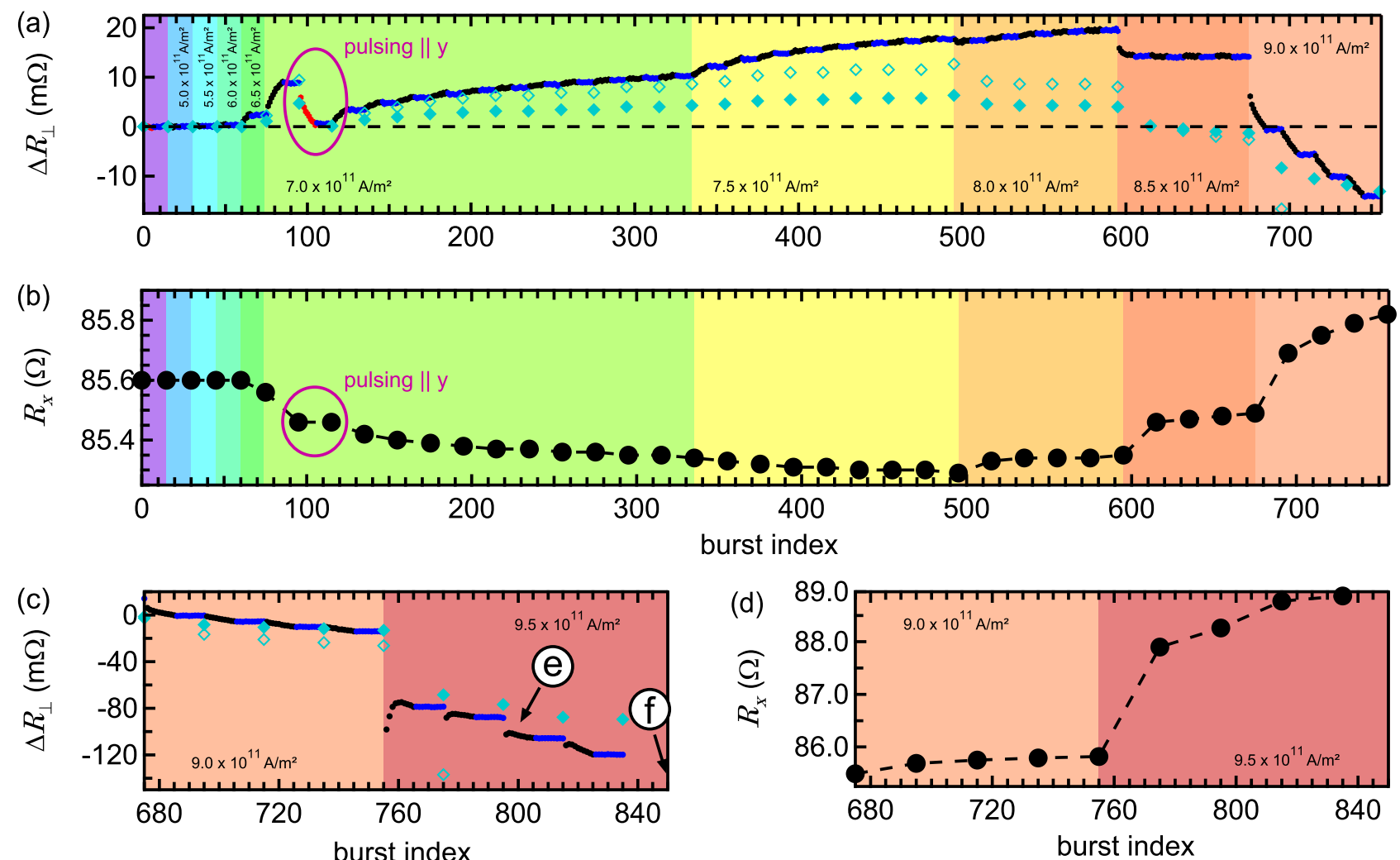

(e)
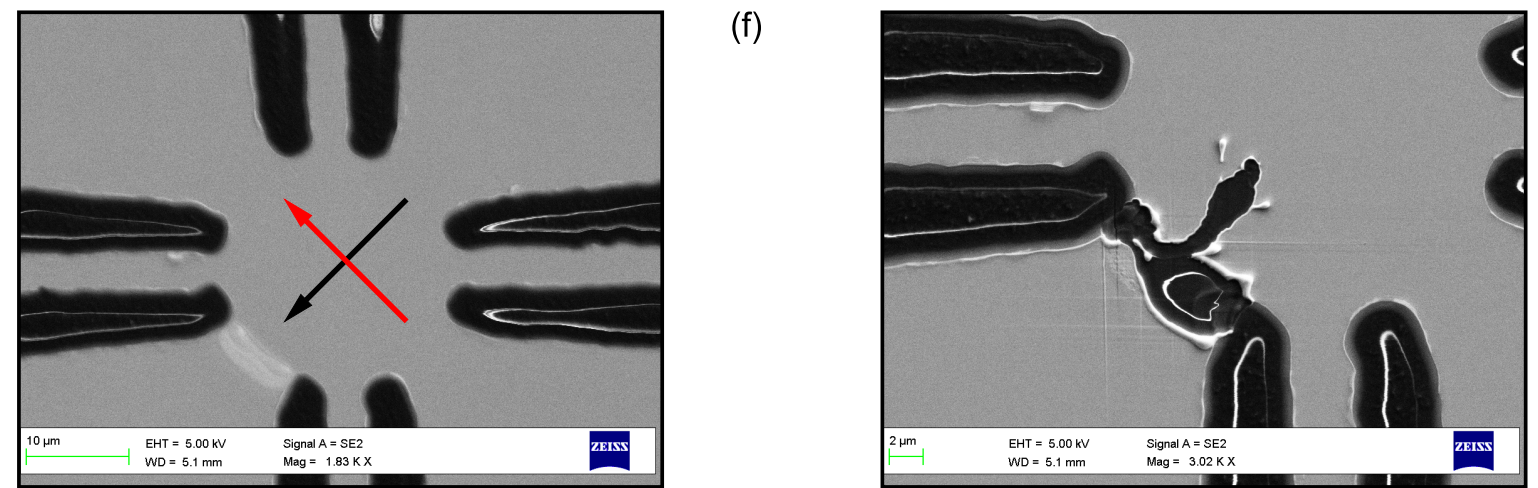

FIG. 3. SEM experiment. Pulses using $\Delta t=10 \mu$ s are applied to a device on the RT sample with $x \| \operatorname{MgO}[110]$. [(a) and (c)] $R_{\perp}$ response to bursts of different $j$. The colors of the data points match the arrows (red, black) in Fig. 1(a) to identify the pulsing direction and the relaxation phase (blue). Filled diamonds in (a) and (c) show the expected $\Delta R_{\perp}$ calculated from $R_{x}$ in (b) and (d) by FEM simulations considering identical resistivity changes in opposing constrictions. The open diamonds are scaled by a factor of two. [(a)-(d)] The background of the plots highlights which magnitude of $j$ is applied. Encircled letters in (c) mark the points at which the SEM images in (e) and (f) have been recorded. [(b) and (d)] $R_{x}$ is measured prior to a pulsing-relaxation sequence. [(e) and (f)] SEM images of the device after the second (e) and fifth (f) repeat with $j \leqslant 9.5 \times 10^{11} \mathrm{~A} / \mathrm{m}^{2}$. The arrows in (e) indicate how the device was contacted [cf. Fig. 1(a)] with the negative polarity for pulsing in $x$ direction in the lower left.

simultaneously specular and off-specular peaks which comes with the cutback that neither peak is measured at optimized diffraction condition. As consequence, the measured intensity is highly sensitive to changes of the samples angles with respect to the beam. The peaks with comparably small error correlate with the movement direction of the stage along the $x$ direction. We reason this by a tiny mechanical instability of the setup, i.e., each time the sample stage moves it tilts slightly with respect to the beam. Due to this technical issue, which is most prominent for movements along $x$ direction, the brightness data scatters significantly more than expected from photon counting statistics. This mechanism has no effect on the measurement performed on the HT sample because $h v$ is optimized solely for the off-specular peaks and, thus, the measured peak intensity is stable with respect to small variations of the samples orientation. We repeat pulsing experiments either until the sign of the response inverts or the device breaks. $j$ is gradually increased to maintain a $\Delta R_{\perp}$ of similar magnitude. For the RT sample, the current density was in the range $j_{\mathrm{RT}}=(7.0, \ldots, 7.8) \times 10^{11} \mathrm{~A} / \mathrm{m}^{2}$ and $j_{\mathrm{HT}}=$ $(7.4, \ldots, 9.0) \times 10^{11} \mathrm{~A} / \mathrm{m}^{2}$ for the HT sample, respectively. We do not observe any electrical response to the beam itself even for long exposure times. We therefore assume that the sample is not altered by the radiation during the experiment. 

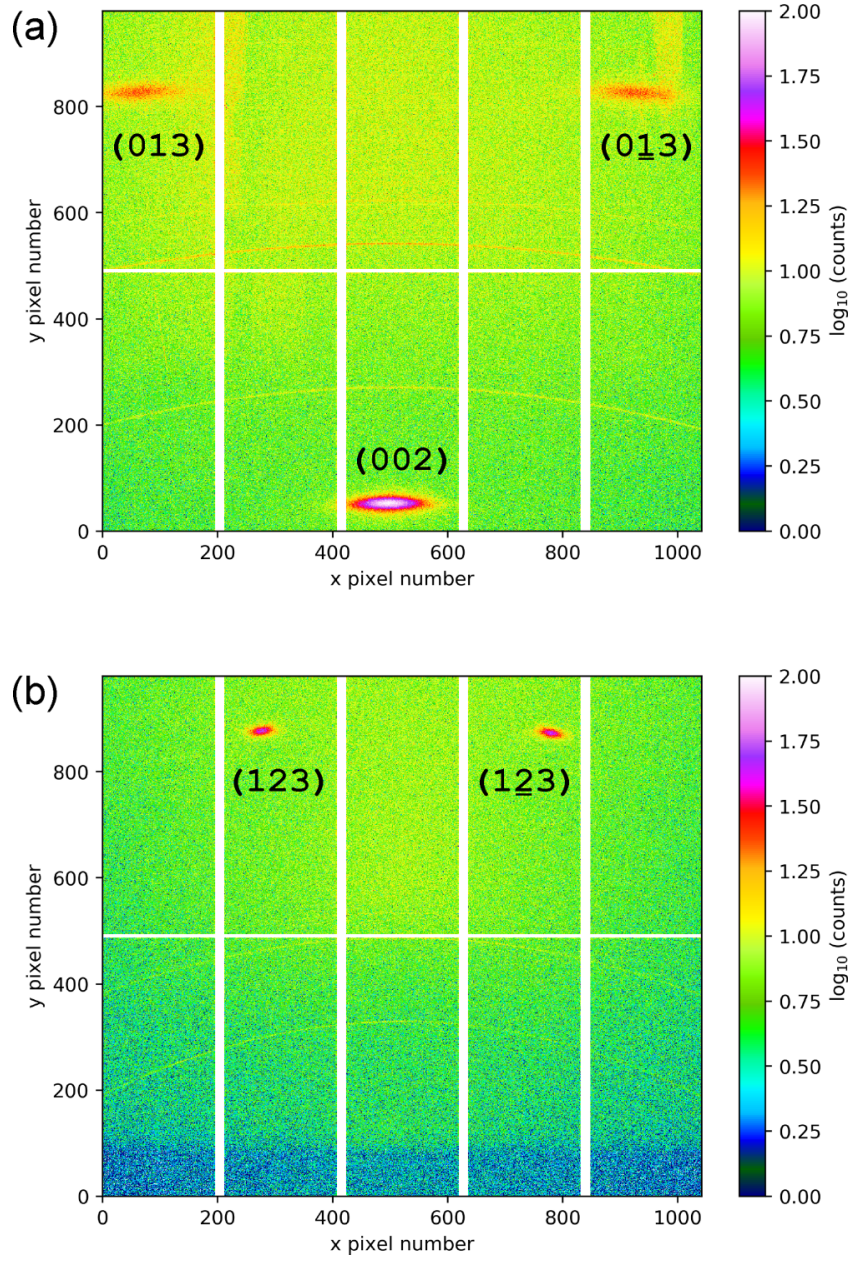

FIG. 4. $\mu$ XRD experiment. Exemplary 2D diffractograms measured with monochromatic x-rays of energy $h v$. The reflexes result from the $\mathrm{Nb}$ film while the rings are due to the $\mathrm{Al}$ bonding wires. (a) RT sample measured with $h v=11.2 \mathrm{keV}$. (b) HT sample measured with $h v=12.6 \mathrm{keV}$. The low-intensity area at the bottom is caused by shadowing from the IC package.

Unfortunately, the signal-to-noise ratio is rather unsatisfying despite the integration times of up to $30 \mathrm{~s}$ per image. Instead of looking at the individual positions, we collect all data sets and apply a linear regression $y=y_{0}+m n$ to extract the trend of peak intensity under repeated electrical pulsing. Each data point is weighted according to its individual error $\Delta I_{n}$ from photon counting statistics. In Fig. 5, these regressions are visualized by the blue lines. We find a positive slope of $m_{\mathrm{RT}}=4.7 \% \circ \pm 0.2 \%$ for the device on the RT sample $\left(y_{0, \mathrm{RT}}=1.000 \pm 0.002\right)$. The fit result is dominated by the data points with comparably low error and, hence, the calculated uncertainty is small as well. The HT sample was destroyed during the experiment with index 7 where pulses were applied in $x$ direction. It can be seen in Fig. 5(c) that the peak intensity measured at the $x$ position after destruction is significantly reduced compared to the other positions. An optical micrograph verifies that indeed the $x$ constriction is the one which broke during the experiment. At the same time, the intensity reduction by only about $20 \%$ demonstrates that
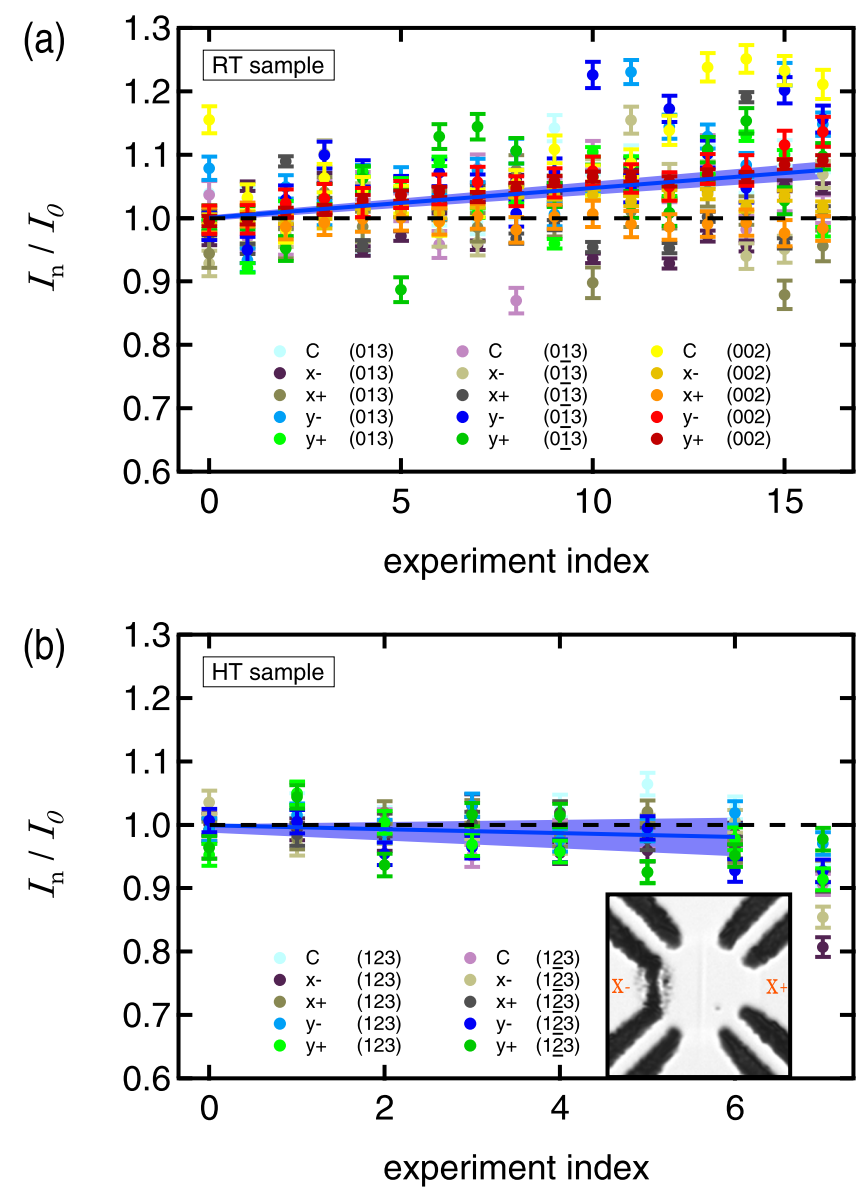

FIG. 5. $\mu \mathrm{XRD}$ evaluation. Plots of the normalized peak intensities $I_{n}$ divided by their values prior to the pulsing experiment for (a) the RT and (b) the HT sample. Error bars are calculated as $\Delta\left(I_{n} / I_{0}\right)=\sqrt{I_{n}} / I_{0}$. The experiment index increases with every pulsing-relaxation sequence. $2 \mathrm{D}$ diffractograms are taken and evaluated for each peak (cf. Fig. 4) and every position on the sample [cf. Fig. 1(a)]. The dashed black lines represent $I_{n} / I_{0} \equiv 1$. Linear fits of the data are shown as blue lines with blue areas representing a $3 \sigma$ surrounding obtained from the fit. The fit in (b) is applied for experimental index $n=0, \ldots, 6$. The inset shows a micrograph of the broken device after $n=7$.

the $\mathrm{x}$-ray spot is much larger than the destroyed area seen in the micrograph. Linear regression with $n=0, \ldots, 6$ gives $m_{\mathrm{HT}}=-3.1 \% \circ \pm 1.1 \%$ and $y_{0, \mathrm{HT}}=0.999 \pm 0.004$. The given uncertainties are the parameter error estimates $\sigma$ obtained from the parameter covariance matrix of the linear fit. We interpret the $\mu$ XRD data as indication for an enhanced crystallinity of the RT sample due to the electrical pulsing. The result for the HT sample aligns with this assumption since no enhancement of the $\mu \mathrm{XRD}$ peak intensity is observed, which is in line with the already good crystallinity [cf. Fig. 1(b)]. One could be even tempted to interpret the HT data as an indication for local destruction of the sample. An increasing pulse line resistance with simultaneous reduction of x-ray diffraction intensity would form a consistent picture. However, the significance of the negative slope of the linear regression is rather weak. 


\section{DISCUSSION}

When a sense current $I_{\mathrm{S}}$ is passed through a material, the appearance of a transverse electric field is generally due to a breaking of the mirror symmetry of the resistivity about the current direction. For the AMR and SMR, this is due to $\rho_{x x} \neq$ $\rho_{y y}$ and the current being neither parallel nor perpendicular to the magnetic moments. As we have shown in this study, one can observe a transverse voltage change induced by electrical pulses that largely resembles the electrical switching of the Néel order in antiferromagnets in devices that are nonmagnetic. Although the amplitude of this nonmagnetic response eventually reduces with successive pulsing, the decay can be marginal and looks like a practically constant amplitude over dozens of repeats.

We interpret our experimental findings on $\mathrm{Nb}$ films as a symmetry breaking on the (macroscopic) device level rather than on the microscopic level. Due to the Joule heating associated with the large current densities, a local variation of the film resistivity $\rho$ is created. This resistivity change $\Delta \rho$ is located in the constrictions of the device where $j$ is large compared to the rest of the device. In the following, we attempt to understand the experimental evidence presented before by a finite-element model. Figure 6(a) shows the locally resolved current density in our eight-arm devices during a pulse. Additionally, we consider another pulse-current distribution in cross-shaped devices, see Fig. 6(c), which we discuss later (cf. Refs. [3,18]).

The large current density in the constrictions of the device was shown to locally change the film crystallinity and to reduce the resistivity as a consequence. For the simplicity of the model, we consider local changes of the resistivity in rectangular areas within the constrictions as depicted in Fig. 6(b). In our example, the dimensions of the pulse line constrictions are about $2 \times 8 \mu \mathrm{m}^{2}$ and each constriction accounts for $\sim 3 \%$ of the total pulse line resistance $R_{x[y]}$. Assuming that $\rho$ only changes within the constrictions, a change $\Delta R_{x}=R_{x}-R_{x, 0}$ can be translated into a resistivity change within the constriction

$$
\frac{\Delta \rho_{\mathrm{c}}}{\rho}=\frac{R_{x} / R_{x, 0}-0.94}{0.06}-1
$$

with $R_{x, 0}$ being the pulse line resistance before pulsing and $\Delta \rho_{\mathrm{c}}$ being small. From the resistance variation of the pulse line in Fig. 3(b), we calculate a maximum $\Delta \rho_{\mathrm{c}} / \rho$ of about $-6 \%$ (around burst index 495). Here, we measure $\Delta R_{\perp}=$ $17.7 \mathrm{~m} \Omega$. We then perform FEM simulations to check whether $\Delta \rho_{c}$ can account for $\Delta R_{\perp}$. The resistivity of the film is set to $\rho_{\mathrm{RT}}$ and the resistivity in the constrictions is $\rho_{\mathrm{a}}=\rho_{\mathrm{RT}}+$ $\Delta \rho_{c}=0.94 \rho_{\mathrm{RT}}$ [cf. Fig. 6(b)]. We then apply $I_{\mathrm{S}}$ and find that a transverse voltage arises due to the symmetry break about the current direction. The simulated transverse resistance is $R_{\mathrm{FEM}}=+8.7 \mathrm{~m} \Omega$. Note that the sign of $R_{\mathrm{FEM}}$ associated with $\Delta \rho_{c}$ depends on the measurement geometry. Throughout the manuscript we refer to the configuration shown in Fig. 1(a). In Fig. 6(a), one can see that the current density distribution in a constriction is inhomogeneous. The highest values of $j$ are located at the device's edges close to the probe lines and also outside of the area we allow for resistivity change. The local
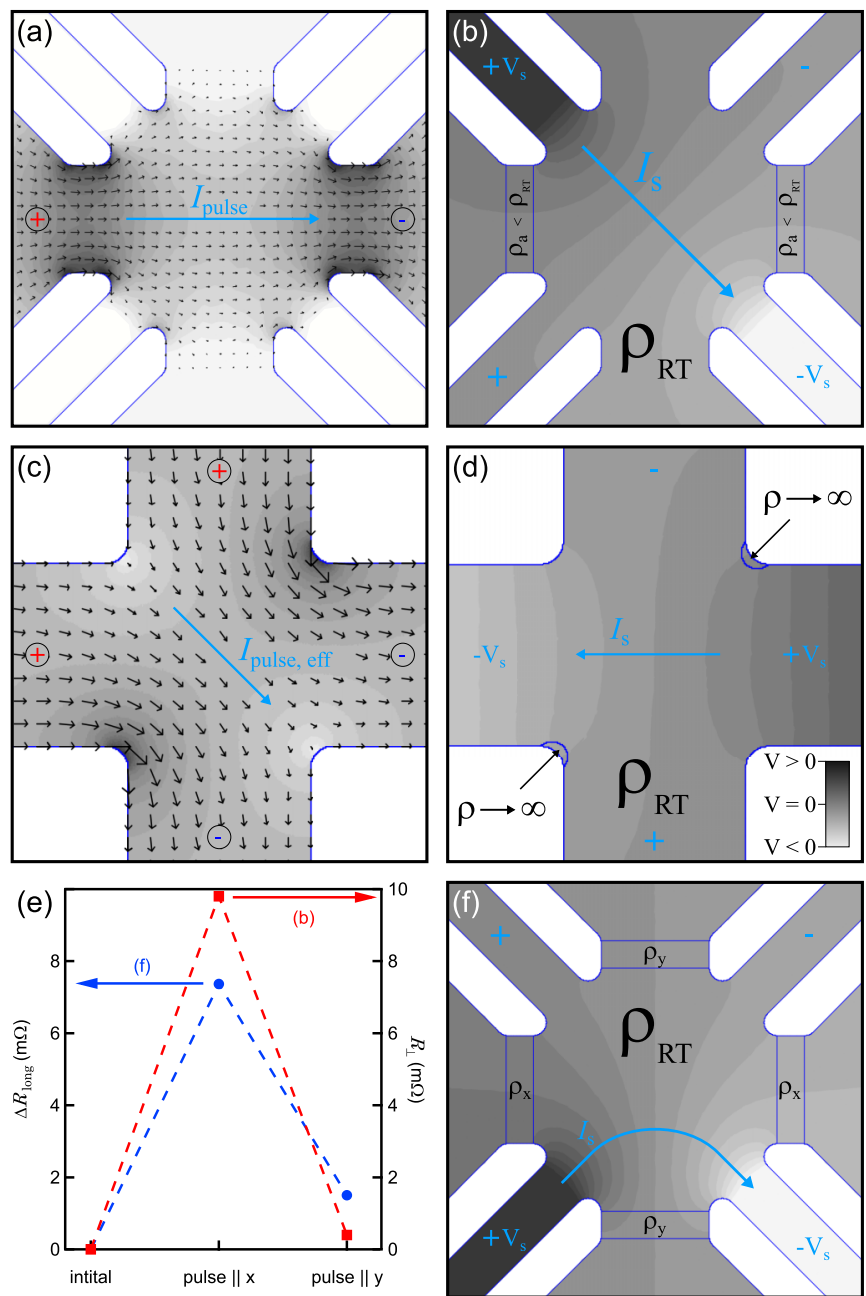

FIG. 6. FEM simulation. [(a) and (c)] Current density distribution during a pulse in (a) star- and (c) cross-shaped devices with homogeneous resistivity. Arrows indicate the conventional current direction. [(b) and (d)] Voltage landscape in devices with locally varying resistivity $\rho_{\mathrm{RT}}, \rho_{\mathrm{a}}$ and $\rho \rightarrow \infty$. (e) Simulation of the pseudolongitudinal resistance change $\Delta R_{\text {long }}$ for the measurement geometry (f) and the $R_{\perp}$ resistance for the measurement geometry (b). Pulsing $\| x$ reduces $\rho_{x}<\rho_{\mathrm{RT}}$ and breaks the symmetry of the device. Pulsing $\| y$ restores the symmetry of the device with $\rho_{y}=\rho_{x}<\rho_{\mathrm{RT}}$. (f) Potential landscape in star-shaped devices using the pseudolongitudinal measurement geometry.

modification of of $\rho$ will be concentrated where $j$ is highest. We therefore attribute the discrepancy $\Delta R_{\perp} \approx 2 \times R_{\mathrm{FEM}}$ to the simplicity of our model, i.e., the particular choice of the rectangular area of resistivity modification. Changing the profile from rectangular to convex or concave and adjusting the resistivity within to match the observed $R_{x}$ has only small effects on $R_{\mathrm{FEM}}$. In Fig. 3(a), we show full calculations with the model using rectangular constrictions, where the local resistivity variation is calculated on the basis of the change in pulse line resistance. We find that the observed transverse resistance is qualitatively well reproduced by the model, although quantitative descrepancies remain, which are probably due to the simplicity of the model. Thus our 
conclusion is that the local change $\Delta \rho_{c}$ in the constrictions of our device contributes largely to the transverse voltage signals. In addition, the sign of the transverse voltage is the same in the FEM calculation and in the experimental lowercurrent density regime of the RT sample. This implies that the sign change of the transverse resistance change in the experiment with the RT sample [cf. Fig. 2(a)] requires a local increase of the resistivity. This is consistent with Figs. 3(a) and $3(\mathrm{~b})$, where the increase of pulse line resistance correlates with a sign reversal in the transverse voltage change. The "saw-tooth"-like electrical response of Figs. 2(a) and 2(b) is explained by considering that for pulsing along one direction the mirror symmetry of the devices local resistivity about $I_{\mathrm{s}}$ is gradually broken with every pulse, whereas pulsing along the orthogonal channel restores the mirror symmetry by also reducing/increasing the resistivity in the other constrictions. We simulated this situation with the FEM model and show the result in Fig. 6(e) (right axis). After restoring the mirror symmetry by reducing the local resistivities along both $x$ and $y$ pulse lines, the transverse voltage drops back to zero within numerical accuracy.

Now we come back to the cross-shaped devices that were used in several previous studies $[3,7,9,18]$. In this configuration, the pulse voltage is applied to pairs of contacts as depicted in Fig. 6(c). Here, the maximum current density is found in the corners of the structure. The readout is done with a simple Hall configuration, see Fig. 6(d). As we lack detailed measurements to compare with, we just show the basic principle of how a transverse voltage could arise in this type of device. With respect to the Hall configuration, the mirror symmetry is again broken by the local resistivity change in the corners and restored by pulsing in the orthogonal direction. In the simulation, we consider $\rho \rightarrow \infty$ for simplicity in a small rounded area close to the corners [Fig. 6(d)]. Clearly, this gives rise to a transverse voltage, which can be seen by the distorted equipotential lines. Quantifying the local resistivity changes in this type of device is probably more difficult than in the star-shaped devices. It does therefore seem advisable to preferably make use of starshaped devices in order to quantify the pulse line resistances for estimating the associated transverse voltage with FEM simulations.

Finally, in some cases a readout configuration as depicted in Fig. 6(f) is used, which we call "pseudolongitudinal." Following previous symmetry-derived arguments, one would not expect an influence of the local resistivity changes. However, our FEM simulation shows that even in this case a change of the pseudo-longitudinal voltage picked up between the top-left and top-right contacts is visible when the constrictions along the $x$ and $y$ directions change resistivity, cf. Fig. 3(e). Here, the reason is that the current-density distribution moves along the $y$ direction, such that more current leaks into the film area between the pickup lines. Due to symmetry, this is not fully reversible by orthogonal pulsing and should lead to a "saw-tooth" signal with a prominent drift given by the inverse sign of the local resistivity change. This drift is readily visible in our simulation in Fig. 6(e). We also note that signal drifts may be ubiquitous as soon as the breaking and restoration of the device symmetry are not perfect, independent of the actual device shape.

\section{CONCLUSION}

We can conclude that we see two different counteracting mechanisms: on the one hand, the resistivity reduction which is probably due to crystallization. On the other hand, there is a mechanism that increases the resistivity and destroys the sample eventually. The annealinglike contribution should not be present in the HT sample which is already of good crystallinity. Consistently, we see only one sign of the transverse voltage change in the HT sample, which corresponds to a local increase of the resistivity according to the FEM simulation. The latter is supposedly related to electromigration, which transports material away from the constrictions, thereby creating voids in the material and increasing the local resistivity. The final destruction of the device goes along with a melting of the film and droplets of the material moving in electron flow direction [cf. Fig. 3(f)]. Electromigration has been investigated extensively decades ago in the course of microelectronics and is a well understood phenomenon [19-22]. It has been identified as the predominant reason for the failure of microstructures under electrical stress. Hence, electromigration is probably the essential mechanism underlying the apparent resistivity increase and eventual breakdown of the device. We note that both the annealing-like component as well as the electromigration-like component are thermally activated and depend on individual energy barriers $Q$ and the temperature $T$ via a Boltzmann factor [23]. In particular, according to Black's equation [24]

$$
\mathrm{MTF}=\frac{A}{j^{2}} \exp \left(\frac{Q}{k_{\mathrm{B}} T}\right)
$$

the mean-time-to-failure (MTF) is a strong function of current density and temperature, where the film temperature itself depends strongly on the current density. Here, $k_{\mathrm{B}}$ is the Boltzmann constant and $A$ is a scaling factor. Due to the high $j$ necessary to switch an AFM, the film temperature may rise by several hundred Kelvin during the pulses [3,4,25]. Thus experiments performed close to the destruction threshold of the devices are particularly prone to the electromigrationlike component of the electrical response and results should be taken with care. In Fig. 7, we evaluate Black's equation within two models, one calculated at fixed $T=300 \mathrm{~K}$, and one calculated at an elevated temperature $T+\Delta T$, where $\Delta T$ is calculated as a pulse-averaged temperature with You's formula [25] for a pulse width of $10 \mu \mathrm{s}$ and typical parameters of the $\mathrm{MgO}$ substrate (see Ref. [3] for details). The parameters for Black's equation are chosen as $Q=0.6 \mathrm{eV}$ and $A=2 \times 10^{17} \mathrm{sA}^{2} / \mathrm{m}^{4}$. While $Q$ is a typical value for grain-boundary self-diffusion [24], $A$ is chosen to roughly match the experimental destruction threshold of the device. It can be seen in Fig. 7(a), that the Joule heating is largely responsible for the short MTF for $j>10^{11} \mathrm{~A} / \mathrm{m}^{2}$. Fig. 7(b) demonstrates that for $j>10^{12} \mathrm{~A} / \mathrm{m}^{2}$, the pulse-averaged film temperature is elevated by more than $700 \mathrm{~K}$ and may reach even higher temperatures in the final stage of device breakdown, when the constriction becomes even more narrow due to the formation of cracks in the hot-spots. This is consistent with the observed droplets in the destroyed device, see Fig. 3(f). In the high-current density regime, Black's equation is often rewritten as $\mathrm{MTF}=A j^{-m} \exp \left(Q / k_{\mathrm{B}} T\right)$ to 

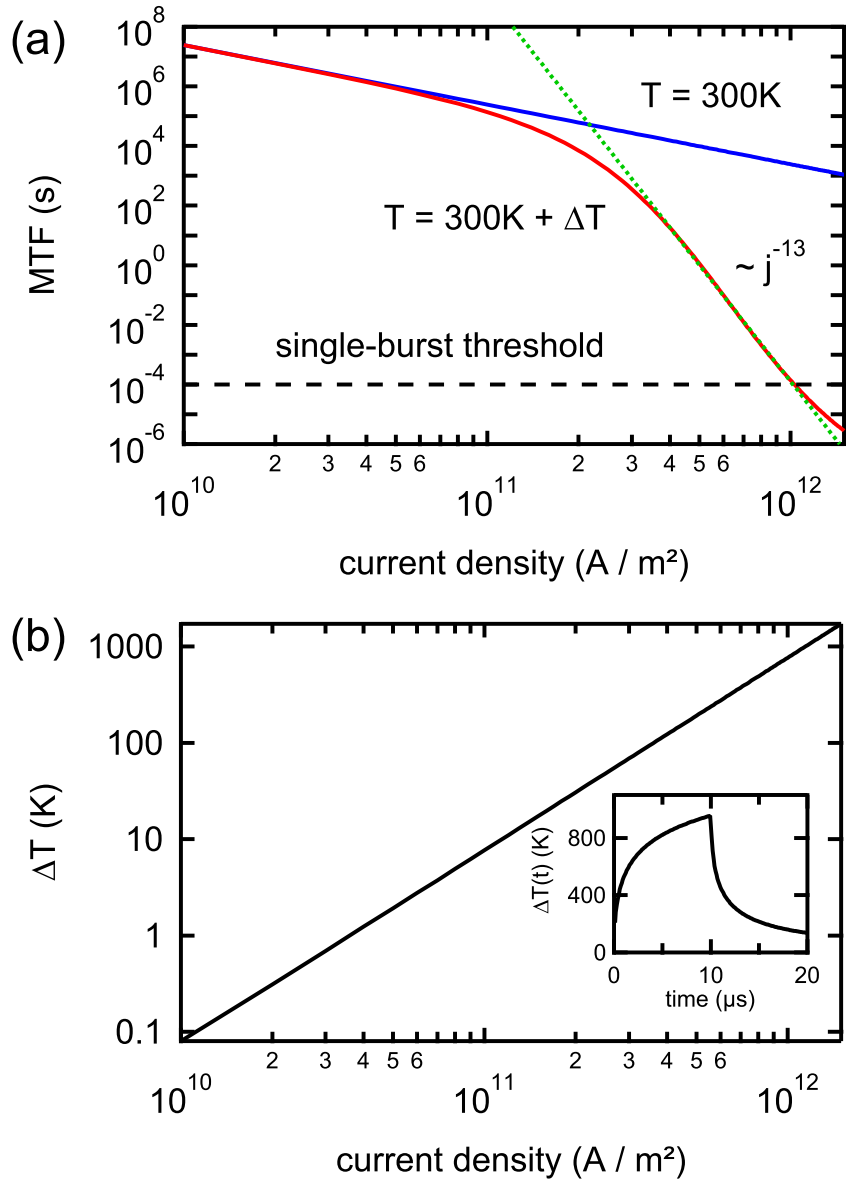

FIG. 7. Black's equation. (a) Mean time to failure (MTF) as a function of the current density in the constrictions of our devices. Two models are considered, one at fixed temperature of $300 \mathrm{~K}$ and one including the Joule heating contribution $\Delta T$. The dotted line represents a power-law fit to the high-current density regime. (b) $\Delta T$ as a function of the current density $j$ calculated with You's formula [25]. The inset shows the time-dependence of the film temperature for a pulse of $\Delta t=10 \mu \mathrm{s}$ and $j=10^{12} \mathrm{~A} / \mathrm{m}^{2}$.

effectively take the Joule heating into account, where $m>2$. Our numerical estimates suggest that $m \approx 13$ in this specific example, i.e., the MTF shows a very rapid decay with increasing $j$. Because of the similarities in the underlying thermal activation physics, we expect that similar dependencies on measurement temperature and current density may occur due to annealing/electromigration and Néel-order switching. Consequently, it is a difficult task to disentangle the various contributions by electrical experiments alone.

However, the FEM model provides means to perform this separation. The basic idea is to estimate the magnitude of $\Delta R_{\perp}$ from the measured longitudinal resistance change $\Delta R_{\text {long }}$ and, thus, conclude whether the annealing/electromigrationlike mechanism does fully account for the observed value. From our experiments we know that we can do this estimation if the result of Eq. (1) is multiplied by an empirically correction factor. Unfortunately, this factor has to be determined for different device geometries individually. Nevertheless, if the factor is known, one can calculate the transverse resistance change $\Delta R_{\perp, \text { FEM }}$ that originates solely from the inhomogeneous resistivity from the measured $\Delta R_{\text {long. }}$. Now one can introduce an anisotropy to the resistivity, i.e., $\rho_{x x} \neq \rho_{y y}$, that may originate from AMR and/or SMR caused by a reorientation of the magnetic order. The corresponding transverse resistance change $\Delta R_{\perp, \mathrm{FEM}}^{\text {ani }}$ can be calculated easily if one assumes a homogeneous resistivity. We adjust $\rho_{x x}$ to match $\Delta R_{\text {long }}$ and $\rho_{y y}$ according to AMR theory. For a magnetically saturated switching, i.e., the constrictions and the center region are switched by a similar proportion, this assumption is reasonable. We find that this leads to approximately $\Delta R_{\perp, \mathrm{FEM}}^{\mathrm{ani}} \approx 10 \times \Delta R_{\perp, \mathrm{FEM}}$. Thus it is clear that an observed transverse response that is larger than what can be accounted for by the resistivity inhomogeneity must be due to a resistivity anisotropy and might originate from the magnetic order.

Using a chemically stable capping layer may reduce the surface contribution to the electromigration. However, the volume contributions of the electromigration can not be reduced by cap layer engineering. As electromigration is a directed transport along the direction of electron flow, one may use single-cycle AC current pulses instead of DC pulses, similar to a previous experiment with CuMnAs [26]. If the current pulses are short enough, this should greatly reduce the directed transport, thereby increasing the device lifetime by orders of magnitude [27]. As consequence, the resistivityenhancing component of the transverse electrical response should be substantially reduced. However, the purely thermal, annealinglike contribution would still be present. This contribution should be relatively easy to remove by pulsedcurrent annealing the device with a larger current density before systematic investigations of the Néel-order switching are performed. In all cases, it is mandatory to carefully monitor the pulse line resistances and the repeatability of an electrical Néel-order switching experiment to avoid possible confusion of the true planar Hall signal due to switching and the resistive contributions.

During this investigation, we did not observe time dependent longitudinal or transversal resistance changes during the relaxation. Hence, we conclude that the temperature of our device is constant in that time, which is in line with You's calculation that predicts a decay of the device temperature within tens of microseconds after the pulse current is switched off [cf. inset of Fig. 7(b)]. Our experiments therefore provide no evidence that supports the presence of lateral temperature gradients seconds after the pulse, which were suggested by Chiang et al. [13]. They also noticed a significant change of the longitudinal resistance in their devices which they do not discuss with regard of its effect on the measured $R_{\perp}$ (cf. Suppl. of Ref. [13]). A model based on thermal gradients can also not explain the positive or negative signs of the transverse voltage that correlate with the pulse line resistance changes. Following our model, this longitudinal resistance change can explain their observation without the assumption of any temperature gradients. We thus conclude that an explanation solely based on thermal gradients and associated thermovoltages is inconsistent with our data. As a final remark, we note that we performed similar experiments with films of Ti, V, Mo, and Ta with various degrees of crystal quality, which support our conclusions. 


\section{SUMMARY}

In summary, we investigated the resistive response of starshaped devices often used in switching experiments with antiferromagnets. We used $\mathrm{Nb}$ thin films of different crystallinity and observed different responses in films with either polycrystalline or epitaxial growth. We reason that a change of the resistivity in the constrictions of the devices leads to a transverse voltage in planar Hall effect measurement geometry. From the pulse line resistance changes, we estimate the magnitude of resistivity change in the constrictions and perform FEM simulations that are in semiquantitative agreement with the experimental data. We attribute the decrease and increase of the local resistivity to a crystallization and destruction of the sample, respectively. In operando SEM imaging and $\mu \mathrm{XRD}$ measurements support our interpretation. The crystallization or annealing effect is not visible in SEM images, whereas $\mu \mathrm{XRD}$ corroborates the hypothesis of local crystallization in polycrystalline films. The sample destruction becomes significant in the transverse and pulse line resistance way before we were able to see any damage in SEM images. This effect resembles the planar Hall effect and is of nonmagnetic origin. Throughout this study, it was found to be irreversible and relaxation was not observed. Thus we think that as long as the electrically set state relaxes, the observed perpendicular resistance change is, at least partially, due to a switching of the magnetic order.

For future electrical switching experiments, it is thus advisable to regularly measure the pulse line resistances and to check the reproducibility of the switching in, e.g., current density sweeps. As the destructive contribution can be practically constant over a vast number of repeats, the reproducibility of the experiment alone without varying parameters and resistance monitoring does not yield sufficient evidence to exclude the discussed nonmagnetic contributions. Furthermore, consistency checks regarding the effect amplitudes using FEM simulations, that can be done with, e.g., the free software FEMM, may help in ruling out possible artifacts. Our intention with this manuscript is to sensitize the community to carefully examine each experimental finding in regard of possible nonmagnetic contributions. We do not judge any already published work regarding electrically induced magnetic switching individually.

\section{ACKNOWLEDGMENTS}

This research used beamline 12.3.2 of the Advanced Light Source, which is a DOE Office of Science User Facility under Contract No. DE-AC02-05CH11231. We further thank Christan Mehlhaff for conducting preliminary experiments.
[1] J. Železný, H. Gao, K. Výborný, J. Zemen, J. Mašek, A. Manchon, J. Wunderlich, J. Sinova, and T. Jungwirth, Relativistic Néel-Order Fields Induced by Electrical Current in Antiferromagnets, Phys. Rev. Lett. 113, 157201 (2014).

[2] P. Wadley, B. Howells, J. Železný, C. Andrews, V. Hills, R. P. Campion, V. Novák, K. Olejník, F. Maccherozzi, S. S. Dhesi, S. Y. Martin, T. Wagner, J. Wunderlich, F. Freimuth, Y. Mokrousov, J. Kuneš, J. S. Chauhan, M. J. Grzybowski, A. W. Rushforth, K. W. Edmonds, B. L. Gallagher, and T. Jungwirth, Electrical switching of an antiferromagnet, Science 351, 587 (2016).

[3] M. Meinert, D. Graulich, and T. Matalla-Wagner, Electrical Switching of Antiferromagnetic $\mathrm{Mn}_{2} \mathrm{Au}$ and the Role of Thermal Activation, Phys. Rev. Appl. 9, 064040 (2018).

[4] S. Y. Bodnar, L. Šmejkal, I. Turek, T. Jungwirth, O. Gomonay, J. Sinova, A. A. Sapozhnik, H.-J. Elmers, M. Kläui, and M. Jourdan, Writing and reading antiferromagnetic $\mathrm{Mn}_{2} \mathrm{Au}$ by Néel spin-orbit torques and large anisotropic magnetoresistance, Nat. Commun. 9, 348 (2018).

[5] X. F. Zhou, J. Zhang, F. Li, X. Z. Chen, G. Y. Shi, Y. Z. Tan, Y. D. Gu, M. S. Saleem, H. Q. Wu, F. Pan, and C. Song, Strong Orientation-Dependent Spin-Orbit Torque in Thin Films of the Antiferromagnet $\mathrm{Mn}_{2} \mathrm{Au}$, Phys. Rev. Appl. 9, 054028 (2018).

[6] X. Z. Chen, R. Zarzuela, J. Zhang, C. Song, X. F. Zhou, G. Y. Shi, F. Li, H. A. Zhou, W. J. Jiang, F. Pan, and Y. Tserkovnyak, Antidamping-Torque-Induced Switching in Biaxial Antiferromagnetic Insulators, Phys. Rev. Lett. 120, 207204 (2018).

[7] T. Moriyama, K. Oda, T. Ohkochi, M. Kimata, and T. Ono, Spin torque control of antiferromagnetic moments in NiO, Sci. Rep. 8, 14167 (2018).

[8] I. Gray, T. Moriyama, N. Sivadas, R. Need, B. J. Kirby, D. H. Low, G. M. Stiehl, J. T. Heron, D. C. Ralph, K. C. Nowack,
T. Ono, and G. D. Fuchs, Spin Seebeck Imaging of Spin-Torque Switching in Antiferromagnetic $\mathrm{Pt} / \mathrm{NiO} / \mathrm{Pt}$ Heterostructures, Phys. Rev. X 9, 041016 (2019).

[9] L. Baldrati, H. Gomonay, A. Ross, M. Filianina, R. Lebrun, R. Ramos, C. Leveille, T. Forrest, F. Maccherozzi, E. Saitoh, J. Sinova, and M. Kläui, Mechanism of Néel Order Switching in Antiferromagnetic Thin Films Revealed by Magnetotransport and Direct Imaging, Phys. Rev. Lett. 123, 177201 (2019).

[10] X. F. Zhou, X. Z. Chen, J. Zhang, F. Li, G. Y. Shi, Y. M. Sun, M. S. Saleem, Y. F. You, F. Pan, and C. Song, From Fieldlike Torque to Antidamping Torque in Antiferromagnetic $\mathrm{Mn}_{2} \mathrm{Au}$, Phys. Rev. Appl. 11, 054030 (2019).

[11] M. Dunz, T. Matalla-Wagner, and M. Meinert, Spin-orbit torque induced electrical switching of antiferromagnetic MnN, Phys. Rev. Research 2, 013347 (2020).

[12] Y. Cheng, S. Yu, M. Zhu, J. Hwang, and F. Yang, Reliable Electrical Switching of Tri-State Antiferromagnetic Néel Order in $\alpha-\mathrm{Fe}_{2} \mathrm{O}_{3}$ Epitaxial Films, Phys. Rev. Lett. 124, 027202 (2020).

[13] C. C. Chiang, S. Y. Huang, D. Qu, P. H. Wu, and C. L. Chien, Absence of Evidence of Electrical Switching of the Antiferromagnetic Néel Vector, Phys. Rev. Lett. 123, 227203 (2019).

[14] T. Matalla-Wagner, M.-F. Rath, D. Graulich, J.-M. Schmalhorst, G. Reiss, and M. Meinert, Electrical Néel-Order Switching in Magnetron-Sputtered CuMnAs Thin Films, Phys. Rev. Appl. 12, 064003 (2019).

[15] M. Kunz, N. Tamura, K. Chen, A. A. MacDowell, R. S. Celestre, M. M. Church, S. Fakra, E. E. Domning, J. M. Glossinger, J. L. Kirschman, G. Y. Morrison, D. W. Plate, B. V. Smith, T. Warwick, V. V. Yashchuk, H. A. Padmore, and $\mathrm{E}$. Ustundag, A dedicated superbend $\mathrm{x}$-ray microdiffraction 
beamline for materials, geo-, and environmental sciences at the advanced light source, Rev. Sci. Instrum. 80, 035108 (2009).

[16] K. Baltzis, The FEMM package: A simple, fast, and accurate open source electromagnetic tool in science and engineering, J. Eng. Sci. Technol. Rev. 1, 83 (2008).

[17] D. C. Meeker, Finite Element Method Magnetics, Version 4.2 (28Feb2018 Build), http://www.femm.info.

[18] K. Olejník, V. Schuler, X. Marti, V. Novák, Z. Kašpar, P. Wadley, R. P. Campion, K. W. Edmonds, B. L. Gallagher, J. Garces, M. Baumgartner, P. Gambardella, and T. Jungwirth, Antiferromagnetic CuMnAs multi-level memory cell with microelectronic compatibility, Nat. Commun. 8, 15434 (2017).

[19] P. S. Ho and T. Kwok, Electromigration in metals, Rep. Prog. Phys. 52, 301 (1989).

[20] D. Pierce and P. Brusius, Electromigration: A review, Microelectron. Reliab. 37, 1053 (1997), reliability Physics of Advanced Electron Devices.

[21] K. Lee, Electromigration recovery and short lead effect under bipolar- and unipolar-pulse current, IEEE International Reliability Physics Symposium (IRPS) Anaheim, CA (IEEE, Piscataway, NJ, 2012), pp. 6B.3.1-6B.3.4.
[22] I. A. Blech and C. Herring, Stress generation by electromigration, Appl. Phys. Lett. 29, 131 (1976).

[23] K. A. Jackson, Kinetic Processes: Crystal Growth, Diffusion, and Phase Transitions in Materials (WILEY-VHC Verlag $\mathrm{GmbH} \&$ Co. KGaA, Weinheim, 2004).

[24] J. R. Black, Electromigration - A brief survey and some recent results, IEEE Trans. Electron Devices 16, 338 (1969).

[25] C.-Y. You, I. M. Sung, and B.-K. Joe, Analytic expression for the temperature of the current-heated nanowire for the currentinduced domain wall motion, Appl. Phys. Lett. 89, 222513 (2006).

[26] K. Olejník, T. Seifert, Z. Kašpar, V. Novák, P. Wadley, R. P. Campion, M. Baumgartner, P. Gambardella, P. Němec, J. Wunderlich, J. Sinova, P. Kužel, M. Müller, T. Kampfrath, and T. Jungwirth, Terahertz electrical writing speed in an antiferromagnetic memory, Sci. Adv. 4, eaar3566 (2018).

[27] B. K. Liew, N. W. Cheung, and C. Hu, Electromigration interconnect lifetime under AC and pulse DC stress, 27th Annual Proceedings., International Reliability Physics Symposium Phoenix, AZ (IEEE, Piscataway, NJ, 1989), p. 215. 